\title{
Efficient Clock Synchronization using Energy Based Proportional Integral and Least Common Multiple Protocol in Wireless Sensor Networks
}

\author{
M. Muthumalathi*, P. B. Pankajavalli and N. Priya \\ Department of Computer Science, Bharathiar University, Coimbatore, India
}

Received 22 December 2018; Accepted 14 July 2019

\begin{abstract}
The packet transmission among sensor nodes mainly focuses on clock synchronization. This paper proposes a protocol for efficient clock synchronization in energy based approaches to overcome the clock synchronization invariances and communication overheads. Clustering technique helps to design the sensor network architectures and broadcast the sensor nodes. The existing Mobility Zonal Based Stable Election Protocol (Mob-ZSEP) protocol identifies best path using clustering approach. The selected best path using Mob-ZSEP routing protocol will be utilized by proposed clock synchronization protocols. This research paper proposes two novel protocols: Energy based Proportional Integral and Least Common Multiple (EPILCM) and Weighted based Least Common Multiple (WLCM). EPILCM protocol hybrids two methods such as Energy based Proportional Integral (EPI) and Energy based Least Common Multiple (ELCM). The proposed EPILCM protocol is used for clock synchronization using energy based approach which reduces energy consumption, synchronization error, propagation delay, clock inaccuracy and communication overhead. WLCM protocol performs transmission based on the weight of the packet in sensor networks. The EPILCM, WLCM protocols are compared and the simulation results shows that EPILCM protocol outperforms WLCM protocol.
\end{abstract}

Keywords: Wireless sensor networks, Sensor nodes, Clock synchronization, Clock parameters, Cluster approaches.

\section{Introduction}

Wireless sensor networks (WSNs) contain small sensing electronic devices which are called as sensors. Sensors are used to monitor, detect and collect physical environmental information [1]. Wireless sensor nodes are usually very small devices which is equipped with a limited energy power. A sensor node is considered as a micro electro mechanical system where each sensor node has deployed in a particular environment for accurately observing physical data [2]. Sensor nodes are mainly used to collect the information and communicate wirelessly to the base station.

The selecting and maintaining the sensor node and routing is a challenging factor in WSNs. so routing techniques are very important for packet transmission [3]. The routing protocols are mainly used for efficient data communication among sensor nodes. The packet transmission in sensor networks depends upon energy of the particular node. Packet will be transmitted with minimum energy if the nodes are located near base station [4]. The packet transmission consumes high energy, and nodes are deployed far away from the base station, so routing techniques are used for data broadcast in WSNs.

Clock synchronization is a main technique for synchronizing the sensor node using the clock in WSNs. The clock synchronization is to provide common synchronization time in the entire sensor network environment [5]. Synchronization performs important roles in a sensor

*E-mail address: muthumalathi3004@gmail.com

ISSN: $1791-2377 @ 2019$ School of Science, IHU. All rights reserved.

doi:10.25103/jestr.124.18 network, such as detection of synchronizing event, data fusion, sleep and wake node performance. If the sensor node clock doesn't synchronize to other nodes it causes packet loss [6]. A synchronization technique considers energy consumption for efficient timing message transmissions. Synchronizing method is used to communicate packet from one sensor node to other sensor nodes, without packet loss and delay [7].

Clock inaccuracy values are changing due to their low cost and low power sensors, environmental conditions, and some network delays occur in a sensor network. To overcome these problems clock synchronization in WSNs is introduced [8]. Clock synchronization algorithms are used to correct, remove or compensate clock parameters in reference clock time in sensor networks. This compensation, correction, adjustments of clock parameters techniques are used to exchanges the information between sensor nodes [9].

Accurate measurement of clock parameters such as clock offset and skew is calculated to achieve the efficient synchronization over the network [10]. Network clock synchronization is used to correct the clock parameters to synchronize the global reference clock time to calculate performance time throughout the sensor network [11]. Clock synchronization protocols uses two schemes: Centralized time synchronization protocol, and Distributed time synchronization protocol. Centralized time synchronization protocol provides poor scalability and robustness of source node to layered topology network. Distributed time synchronization protocol depends on clock information; it helps to improve the clock parameters [12]. 
Table 1. Few clock parameters in WSNs

\begin{tabular}{l|l}
\hline Clock parameters & Descriptions \\
\hline Clock offset & $\begin{array}{l}\text { Time variation in synchronizing } \\
\text { two sensor nodes. } \\
\text { Frequency-time variation between } \\
\text { synchronizing two sensor nodes is } \\
\text { called clock skew [13]. } \\
\text { Many sensor nodes clock value } \\
\text { mismatches the reference clock } \\
\text { value. } \\
\text { The time difference of ideal sensor } \\
\text { node clock value. } \\
\text { Sensor nodes time event is recorded } \\
\text { by a particular machine [14]. }\end{array}$ \\
\hline
\end{tabular}

\subsection{Clock Parameters in WSNs}

Clock parameters are difficult to maintain in environmental conditions [15]. There are many parameters in clocks such as offset, skew, jitter, timestamp and drift. Tab 1 describes the clock parameter in WSNs.

Each sensor node has a unique clock, that clock will be considered as $\mathrm{C}_{\mathrm{i}}$. The clock offset is considered as $(\mathrm{O})$ and clock skew as $(\mathrm{S})$. Here initial clock value will be considered as $\mathrm{x}_{\mathrm{i}}(0)$ given by Eq. 1 . The Eq. 1 is derived from local hardware clock equation [16]. The $\mathrm{x}_{\mathrm{i}}(\mathrm{t})$ is the unique clock value of a sensor node $C_{i}$.

$x_{i}(\mathrm{t})=s_{x i}(0)+0$

Clock offset is the time difference between two clocks. Here assume that all the clocks were initially set to a unique clock time $\mathrm{x}_{\mathrm{i}}(0)$ at $\mathrm{x}=0$. Every clock value will be a change in physical and environmental conditions such as temperature, humidity, and vibrational clock (eg. Vehicles clock). The time difference between two clock value is clock offset(O) and the frequency time difference between two clock value is clock skew(S).

Here considering two sensor nodes $\mathrm{x}$ and $\mathrm{y}$, it has own clock value denoted by $\mathrm{x}_{1}(0)$ and $\mathrm{x}_{2}(0)$ for frequencies and Phase of clock value denoted by $\mathrm{y}_{1}(0)$ and $\mathrm{y}_{2}(0)$.

Considering $\mathrm{x} 1(0)$ and $\mathrm{x}_{2}(0)$ have same frequencies [12]

$S_{1} x_{1}(0)=S_{2} x_{2}(0)$

For example considering two sensor nodes have different frequencies

$S_{1} x_{1}(0)<S_{2} x_{2}(0)$ and $S_{1} x_{1}(0)>S_{2} x_{2}(0)$

Considering $\mathrm{y}_{1}(0)$ and $\mathrm{y}_{2}(0)$ have same phases

$O_{1} y_{1}(0)=O_{2} y_{2}(0)$

For example considering two sensor nodes have different phases

$O_{1} y_{1}(0)<O_{2} y_{2}(0)$ and $O_{1} y_{1}(0)>O_{2} y_{2}(0)$

The clock offset of two sensor nodes will be same, and then the clock skew is considered for accurate time calculation. If there is a difference in clock offset and skew of a sensor node then the nodes have a synchronization error where packet delay will occur [17].

This paper discussed about the two proposed protocols WLCM and EPILCM. The proposed WLCM clock synchronization protocol performs weighted based approach to synchronize the packet among sensor nodes. The existing system focused mainly on weighted based packet transmission whereas energy is not considered. So to incorporate an energy based approaches, EPILCM protocol is proposed for efficient communication using clock synchronization. The EPILCM protocol has two methods: i) EPI method which is used to compensate the clock parameters and ii) ELCM method is used to synchronize the clock to the base station.

The proposed EPILCM clock synchronization protocol is used to calculate the propagation delay, synchronization error, clock parameter and communication overhead of the entire sensor networks.

\section{List of Abbreviations}

$\begin{array}{ll}\text { WSNs } & \text { Wireless Sensor Networks } \\ \text { EPILCM } & \text { Energy based Proportional Integral and } \\ \text { Least Common } & \text { Multiple } \\ \text { EPI } & \text { Energy based Proportional Integral } \\ \text { ELCM } & \text { Energy based Least Common Multiple } \\ \text { WLCM } & \text { Weighted based Least Common Multiple } \\ \text { CH } & \text { Cluster Head } \\ \text { Mob-ZSEP } & \text { Mobility-Zonal based Stable Election } \\ \text { Protocol } & \\ \text { EBP } & \text { Estimator-Based Protocol } \\ \text { ATSP } & \text { Average Time Synchronization Protocol } \\ \text { CSLCM } & \text { Clock Synchronization using Least } \\ \text { Common Multiple } & \end{array}$

\section{Related works}

This section describes the existing clock synchronization protocols and algorithms in WSNs. Many researchers focus on clock synchronization problems in a centralized system and distributed system [18]. These systems face many problems of synchronizing sensor nodes, clock parameter invariance, and delay of the communication systems. The existing clock synchronization protocols and methods are mainly contributed to receiver-receiver synchronization, sender-receiver synchronization and two-way message exchange in the network [19].

The existing proportional integral Estimator-Based Protocol (EBP) for clock synchronization can achieve high synchronization accuracy under time-varying clock parameter over the sensor network. This work considers the stability property of EBP and analyses the convergence property under both delay-free and random delay cases. And pseudo-synchronous is used for restricting synchronization error of clock skew into a relatively small quantity which could reduce periodic re-synchronization frequencies [20].

In existing approaches, clock synchronization, average time synchronization provides the average time for the local clock time of the entire network. Average time synchronization means each node has to synchronize to their average value; it gives better scalability and robustness of the network. Average Time Synchronization Protocol (ATSP) is used to share the local clock time for the entire network. So it compensates both clock offset and clock skew value [21].

In some of the existing protocols, all nodes synchronize to their reference clock value. In clock synchronization virtual clock or hardware clock value is modified to achieve the best synchronization. The propagation delay occurs when timing message is communicated among nodes. In Clock Synchronization using Least Common Multiple (CSLCM) 
protocol, each sensor node synchronizes the time period. The CSLCM method does not change the hardware clock time because this protocol does not have a reference clock in base station, so synchronization error value is zero. When synchronization value is zero, the clock offset and skew will not be considered [22]. In CSLCM method, propagation delay will be constant so clock parameter will be removed while packet transmission. It is consensus-based synchronization so Least Common Multiple (LCM) value will be applicable for synchronization among the sensor nodes. LCM of clock period value is calculated for both the inter-cluster and intracluster synchronization. Clock synchronization is used for weight averaging function and it utilizes the minimum synchronization error and fast convergence.

\section{Methodology}

The proposed clock synchronization protocol performs effective synchronization among packet transmission in sensor networks. This section describes the proposed EPILCM clock synchronization protocol and WLCM clock synchronization protocol.

\subsection{Synchronization route selection}

The existing Mobility-Zonal based Stable Election Protocol (Mob-ZSEP) protocol is used to select the best path and the Mob-ZSEP protocol [23] will be used by EPILCM protocol. The best route selection based on sensor node deployment. Fig 1 shows the sensor nodes deployment.

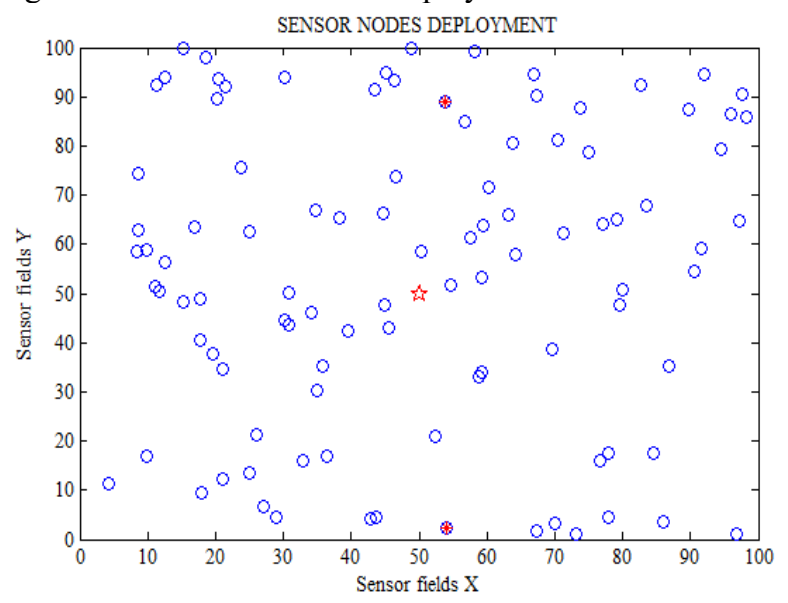

Fig 1. Sensor nodes deployment

\section{A. Node creation}

The sensor node is randomly deployed in the network; it contains $\mathrm{N}$ nodes in $\mathrm{X} * \mathrm{Y}$ network fields. Sensor nodes consist of the cluster member node, cluster head node for sensing data efficiently and to communicate with the base station. Sensor nodes are classified into two levels such as normal node and advanced node. The normal node is provided with minimum level energy for its operation, whereas advance nodes have extra energy than normal node as it consumes less amount of energy for processing.

\section{B. Cluster Setup}

Cluster formation in sensor nodes are divide into three zone levels such as zone 0 , zone 1 and zone 2 which depends on the base station. Normal nodes are deployed in zone 0 level, it placed between $30<\mathrm{Y}<=80$ network fields. Advanced nodes are deployed in zone 1 , zone 2 level, it is placed between $0<\mathrm{Y}<=30,80<\mathrm{Y}<=100$ network fields respectively.

\section{Cluster Head (CH) selection}

The $\mathrm{CH}$ selection is based on maximum energy of a sensor node and it sense the data transmit to the base station. The advanced node which has maximum energy will be considered as $\mathrm{CH}$ and normal node communicates directly to the base station. Normal node energy consumption depends on the distance among the node from the base station. Advanced node senses data and sends the data to $\mathrm{CH}$, where $\mathrm{CH}$ aggregates the data and sends to the base station.

\section{Sensor node delay calculation}

Energy efficient clock synchronization helps to reduce the number of transmission message among synchronizing sensor nodes. This energy consumption procedure is divided into two phase, such as discovery phase and synchronization phase. In the discovery phase, nodes are assigned in a hierarchal level. Once the discovery phase completes its process then the synchronization phase will start. Here synchronization phase is used to communicate among sensor nodes. Some propagation delays may occurs in clock synchronization among the sensor nodes. The EPILCM clock synchronization protocol performs efficient synchronization for the entire sensor networks.

Here it first calculates the propagation delay for the synchronization route. This propagation delay is calculated mainly for compensating the clock inaccuracy in an efficient manner. Clock synchronization parameter variances are considered based on the Eq. 3 and 4. The Eq. 5 is delay calculation for two- way message exchanges in sensor nodes

The time delay calculation [5] in a sensor network is

Delay $=\left(t_{4}-t_{1}\right)-\left(t_{3}-t_{2}\right) / 2$

Base station clock time is considered as $t_{3}, t_{4}$ in sensor nodes and $t_{1}, t_{2}$ time is an acknowledgment reply from base stations.

The reference clock time is used to calculate the delay time of sensor nodes. Here reference clock time is base station clock time. The current time of base station time is denoted as $\mathrm{BS}_{\text {time, }}$, it is used to calculate the sensor node delay time in the entire sensor network. The $\mathrm{CH}_{\mathrm{dt}}$ is a $\mathrm{CH}$ delay time, Eq. 6 is applied to calculate the $\mathrm{CH}$ delay time between the synchronized sensor nodes. Eq. 6 is used to calculate the entire sensor network time delay.

$\mathrm{CH}$ time delay calculation in a sensor network is

$C H_{d t}=B S_{\text {time }}-$ Delay

The propagation delay calculation is used to calculate the synchronization clock inaccuracy among communicating nodes. After calculating the propagation time delay in sensor node, the synchronization error for compensating the clock inaccuracy is compared with the reference clock time.

\subsection{Energy Based Proportional - Integral and Least Common Multiple (EPILCM)}

Clock synchronization protocols in WSNs have less energy consumption and hard to calculate the accurate clock parameters values and also it has message overheads in communicating via the sensor nodes. To overcome these clock synchronization problems, EPILCM protocol was proposed in terms of synchronization accuracy, energy efficiency, and complexity. Fig 2 Illustrate flowchart shows the EPILCM protocol performance.

In this section proposed EPILCM clock synchronization protocol which consists of number of modules such as best 
route selection, energy efficiency, EPI method and ELCM method are discussed.

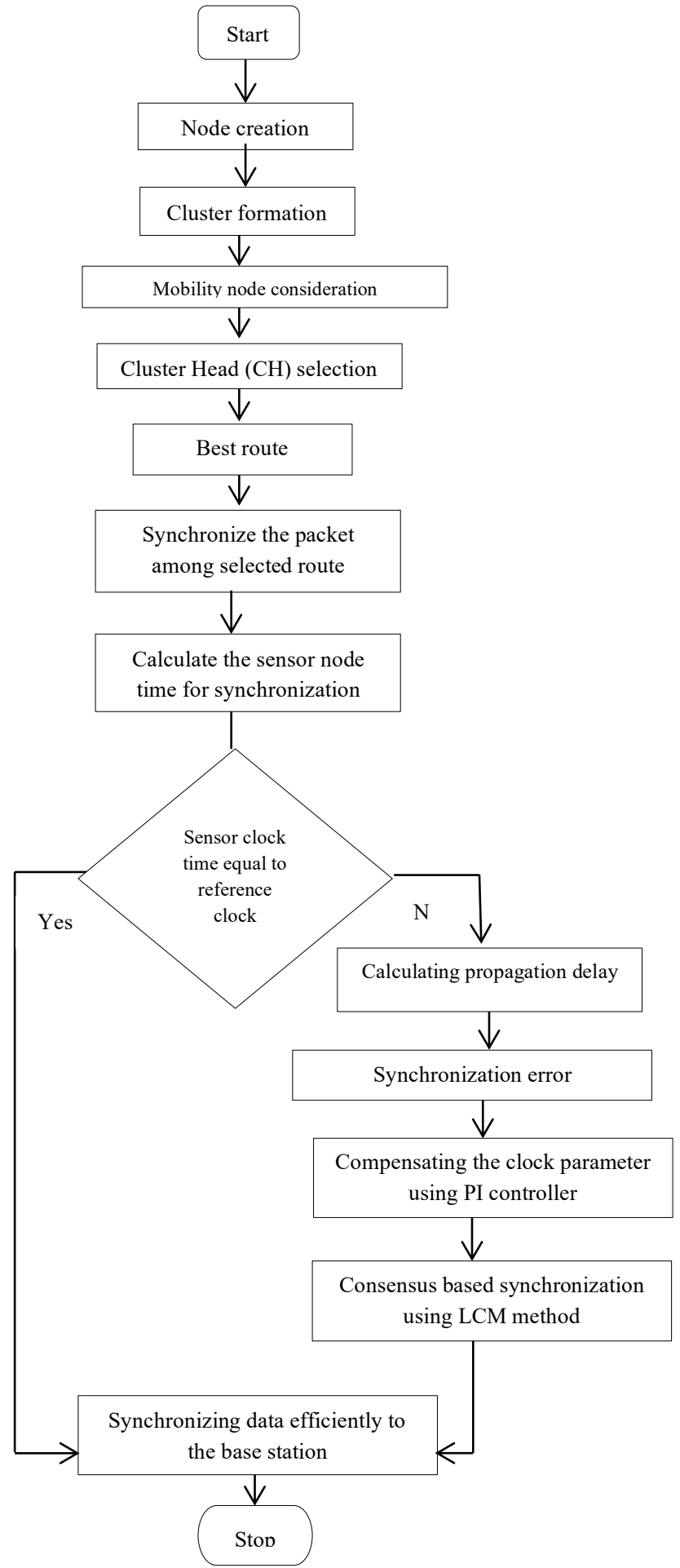

Fig 2. Illustration of EPILCM Protocol

Clock synchronization methods are mostly based on exchanging clock information between the sensor nodes. While exchanging the message, some propagation delay and synchronization error will occur. Most of the synchronization methods in WSNs are used to correct the local clock value of each sensor nodes as a reference clock value. In sensor networks each node transmits packets, while transmitting the packets clock inaccuracy will occur based on physical phenomena and it's difficult to predict and compensate the synchronization error and clock parameter value. Eq. 7 represents general form of sensor node clock function.
Sensor node clock function $C(t)=0+S . t$

Two nodes between clock function node $\mathrm{x}$ and node $\mathrm{y} . \mathrm{t}$ is a node time and Eq. 8 is used to calculate the sensor node clock time.

$C_{y}(t)=O_{x y}+S_{x y} . C_{x}(t)$

Eq. 9 is used to calculate the sensor node clock offset value. $t_{1}, t_{3}, t_{4}$ and $t_{2}$ is represent sensor nodes time.

Offset $O=\frac{\left(t_{2}-t_{1}\right)+\left(t_{4}-t_{3}\right)}{2}$

Eq. 10 is used to calculate the propagation delay.

Propagation delay $\mathrm{d}=\frac{\left(t_{2}-t_{1}\right)-\left(t_{4}-t_{3}\right)}{2}$

Here $T_{2}$ is considered as receiver time and $T_{1}$ considered as sender time. Eq. 11 is used to calculate the senor node receiver clock value. $T_{x y}$ is a node $x$ clock value when receiving broadcast message. $T_{\mathrm{yk}}$ is a node y clock value when receiving broadcast message.

$T_{2}=T_{1}+d-O$

Clock offset $O_{x y}=\frac{1}{\mathrm{n}} \sum_{\mathrm{k}=1}^{\mathrm{n}}\left(T_{x k}-T_{y k}\right)$

Eq. 12 is used to calculate the clock offset error. Here $\mathrm{x}$ and $\mathrm{y}$ are two receiver, $\mathrm{n}$ is a number of broadcast message.

Clock skew error value is described in $(\mathrm{k}-1)$. Clock skew value is derived from two nodes when delay $(i, j)$ occurred in transmission process. Eq. 13 is used to calculate the sensor node clock skew error.

Clock skew error $\mathrm{Si}=\frac{x j\left(t_{k}\right)-x j\left(t_{k-1}\right)}{x i\left(t_{k}\right)-x i\left(t_{k-1}\right)}$

Eq. 14 is used to calculate the clock synchronization error.

Clock synchronization error $C_{y}(\mathrm{t})=O_{x y}+S_{x y} \cdot C_{x}(t)$

Eq. 15 is derived from Eq. 12 and 13.

$\mathrm{Cy}(\mathrm{t})=\frac{1}{\mathrm{n}} \sum_{\mathrm{k}=1}^{\mathrm{n}}\left(T_{x k}-T_{y k}\right) \frac{x j\left(t_{k}\right)-x j\left(t_{k-1}\right)}{x i\left(t_{k}\right)-x i\left(t_{k-1}\right)} . C_{x}(\mathrm{t})$

Eq. 16 is derived from Eq. 15 and $\Delta$ is considered as clock offset error.

$C_{y}(\mathrm{t})=\frac{1}{\mathrm{n}} \sum_{\mathrm{k}=1}^{\mathrm{n}} \Delta+\frac{x j\left(t_{k}\right)-x j\left(t_{k-1}\right)}{x i\left(t_{k}\right)-x i\left(t_{k-1}\right)} \cdot C_{x}(\mathrm{t})$

Eq. 17 is used to calculate the LCM for before compensating synchronization error.

Clock value $\mathrm{C}(\mathrm{t})=\operatorname{lcm}\left(x_{t 1}, y_{t 2}\right)$

PI method applied in Eq. 18 and 19. These Equations are used to compensate the synchronization error. $\mathrm{e}_{\mathrm{y}}(\mathrm{t})$ is a synchronization error compensation. 


$$
e_{y}(\mathrm{t})=t_{y}(\mathrm{t})-t_{x}(\mathrm{t}) \cdot \mathrm{x}(\mathrm{t})
$$

Synchronization error for each clock time interrupt is considered as i, j.

$$
e_{y}(t)=K_{i} \int e_{j}(\mathrm{t}) \mathrm{dt}-K_{p} e_{i}(\mathrm{t})
$$

Adjustment of the clock offset and clock skew value is used for efficient synchronization over the sensor network. Eq. 20 used to calculate the LCM for after compensating synchronization error.

Clock value $\mathrm{C}(\mathrm{t})=\operatorname{lcm}\left(x_{t 1}, y_{t 1}\right)$

The proposed EPILCM protocol allows the nodes to attain the clock synchronization time by calculating propagation delay, compensating the clock offset and clock skew of the entire network.

3.2.1. Energy Based Proportional - Integral (EPI) method The EPI method is used to compensate the invariance of synchronization clock error time in sensor nodes. The synchronization error is mainly considered for efficient clock synchronization in WSNs. This proposed method is used to compensate the clock parameters based on PI-Controller. In EPI, proportional denotes for (P) and integral denotes for (I). $\mathrm{P}$ is used to compensate the clock offset inaccuracy and $\mathrm{I}$ is used to compensate the clock skew inaccuracy in the sensor networks. The aim of the proposed protocol is to reduce the synchronization error over the network.

This section, describes the EPI protocol performance for calculating inaccuracy and compensating the clock parameter. Initially the synchronization error will be constant for the sensor node. After calculating the propagation delay, the delay will be considered as a synchronization error. For compensating the synchronization error EPI protocol were used.

The synchronization error period initially is considered as constant. Synchronization error will be calculated using sensor target value and sensors reading value. For example actual packet receiving time is 12:00:00 and sensor reading time for received packet time is $11: 58: 43$ or 12:08:23, then it indicates there is a change due to environmental conditions and propagation delay occurrence. Eq. 21 is applied for calculating synchronization error.

\section{Synchronization error $=$ Target time - Sensor reading time}

After calculating the synchronization error, the EPI method is performed for compensating the clock invariance. Eq. 22 is formula of Proportional Integral

$P=P+I(1 / S)$

The proportional method is used to compensate the clock offset for synchronized nodes. The clock offset calculation is done using Eq. 23. The Proportional method, $\mathrm{K}_{\mathrm{p}}$ is denoted as coefficient of proportional value.

$$
\text { Proportional }=K_{p} * \text { Synchronization error }
$$

The integral method is used to compensate the clock skew for synchronized nodes. The integral method is used to calculate the clock skew. The clock skew calculation is done using the integral method based on Eq. 24 where $\mathrm{K}_{\mathrm{i}}$ is denoted as coefficient of integral value.

Integral $=K_{p} *$ Synchronization error $+K_{i}$

Here, to calculate the accurate clock value, and to compensate the clock value, $\mathrm{P}$ and I parameters are used. This approach is based on energy for calculating the inaccuracy of clock offset and clock skew.

The clock parameter invariances compensation is mainly used to consensus the synchronization over the entire network. The EPI method reduces the clock invariance in the sensor networks. The clock parameters compensation is performed based on the consensus synchronization.

\subsubsection{Energy Based Least Common Multiple (ELCM) method}

ELCM method is used to transmit the packet over the sensor nodes for efficient synchronization. Clock synchronization is an important fact in sensors for sending the packet to the base station. The clock will be focused when this sensor senses, monitors and detects the data constantly and periodically communicate to the appropriate environment. Here Least Common Multiple (LCM) method is used for energy based consensus clock synchronization approach. For better synchronization of the packet transmission to the base station; among the sensor node the clock parameter values are compensated before and after using LCM.

Considering the compensated value for efficient transmission in the sensor nodes, clock parameters value is denoted as $\mathrm{C}_{\mathrm{BC}}$ before compensating clock parameter values and $\mathrm{C}_{\mathrm{AC}}$ denote the clock after compensating clock values.

Synchronization time $=\operatorname{lcm}\left(C_{B C}, C_{A C}\right)$

The synchronization messages are caused by some delay, this delay will occur before it reaches the destination. The ELCM method is mainly used to send packets to the base station at correct clock time. Eq. 25 is used to communicate among sensor nodes for efficient consensus based synchronization.

The proposed EPILCM protocol is used to reduce the propagation delay, clock parameter inaccuracy error and communication overhead among the sensor node in the entire network and also the clocks are synchronized, which reduces packet loss and reliability in sensor network.

\subsection{Weighted Based Least Common Multiple (WLCM)}

The weighted based approaches are used for clock synchronization in sensor network, which achieve synchronization over the network. This protocol does not consider energy techniques and clock parameters are not compensated during packet communication. The propagation delays are sometimes constant for adjusting the clock rate, which leads clock inaccuracy to be minimum while packet transmission. This method was performed using weighted based approaches for packet transmission. Fig 3 shows the flowchart of WLCM protocol performance.

The node $\mathrm{x}$ is transmitting the synchronization message and it has the local timestamp denoted as $\mathrm{T}_{\mathrm{x}}(\mathrm{t})$, the timestamped value is compared to reference clock value which is denoted as $\mathrm{C}_{\mathrm{x}}(\mathrm{t})$. The node $\mathrm{x}$ transmits the message, delay will occur while packet synchronization. The synchronization error will be calculated and compared with the sender time to their own reference clock time. Receiver node y observe the arrival of local clock time value which is denoted as $\mathrm{T}_{\mathrm{y}}(\mathrm{t})$. 
This value is compared as the reference clock time $\mathrm{C}_{\mathrm{y}}(\mathrm{t})$. The arrival time stamp value is subtracted from the own reference clock time to adjust the neighbor node time.

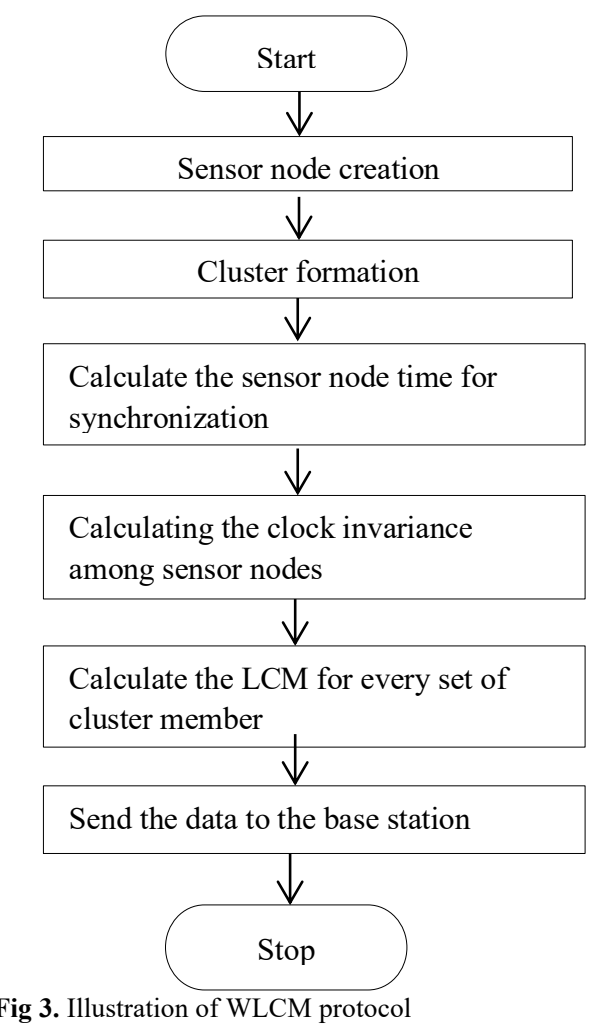

The WLCM protocol has some difficulties for efficient packet transmissions in sensor networks. This protocol does not consider any reference clock time for accurate time calculations and residual energy for packet transmission. It is performed based on the weight of the packet which is used for the transmission in sensor networks. The sensor nodes clock time synchronization rarely calculates the synchronized packet. To overcome these difficulties EPILCM is proposed for clock synchronization in sensor networks.

Here the EPILCM synchronization protocol and WLCM synchronization method is calculated for the accurate clock offset and skew difference, synchronization error variance, propagation delay time and packet communication overheads.

\section{Simulation Result and discussion}

This section discusses the analysis and design of EPILCM protocol and WLCM protocol. This simulation result shows the performance of EPILCM protocol and WLCM protocol in terms of clock parameter invariance, synchronization error, communication overheads, and propagation delay.

The proposed EPILCM was simulated using MATLAB and SIMULINK. Here 100 sensor nodes are considered in a sensor network fields. Tab 2 shows the simulation parameter of EPILCM protocol.

Table 2. Simulation parameters of EPILCM

\begin{tabular}{l|l}
\hline Parameters & Values \\
\hline Number of sensor nodes & 100 \\
Number of rounds & 9000 \\
Deployment of sensor nodes & Random \\
Sensor field environment & $100^{*} 100 \mathrm{~m}$ \\
Initial Energy $\left(\mathrm{E}_{0}\right)$ & $0.5 \mathrm{~J}$ \\
\hline
\end{tabular}

\begin{tabular}{l|l}
\hline $\begin{array}{l}\text { Advanced nodes initial energy } \\
\text { The Energy of Data collection }\left(\mathrm{E}_{\mathrm{DA}}\right)\end{array}$ & $\mathrm{E}_{0}(1+\mathrm{a})$ \\
$\begin{array}{l}\text { Probability }(\mathrm{p}) \\
\end{array}$ & 0.1 \\
\hline
\end{tabular}

The EPILCM clock synchronization protocol is used efficiently for synchronizing among two sensor nodes and to transmit packet to the base station efficiently. The EPILCM protocol calculates the synchronization error of different nodes and communications is done between the nodes.

Simulation results compare the performance of EPILCM with WLCM at the network level. The proposed EPILCM performs better than the WLCM. Tab 3 shows the simulation parameter of WLCM protocol.

Table 3. Simulation parameters of WLCM

\begin{tabular}{l|l}
\hline Parameters & Values \\
\hline Number of sensor nodes & 100 \\
Number of rounds & 9000 \\
Deployment of sensor nodes & Random \\
Sensor field environment & $100^{*} 100 \mathrm{~m}$ \\
Size of the packet & 500 Byte \\
\hline
\end{tabular}

The simulation result discusses the synchronization error variance during the probability of the packet loss. The below clock synchronization figures show the comparison of EPILCM and WLCM protocols. The figures $(4-8) \mathrm{x}-$ axis denotes the time series and $y$ - axis denotes the signal values.

Clock synchronization delay may occur while transmitting the packet. The Propagation delay is calculated in network synchronization and propagation delay is calculated when packet transmission is done to compensate the clock parameter performance. The EPILCM propagation delay signal value ranges from 0 to $23204 \mathrm{e}^{-04}$ and WLCM propagation delay signal value ranges from 0 to $-1.1602 \mathrm{e}^{-04}$. The sensor nodes propagation delay calculations are based on equation 6. Fig 4 shows the propagation delay comparisons of EPILCM and WLCM protocols.

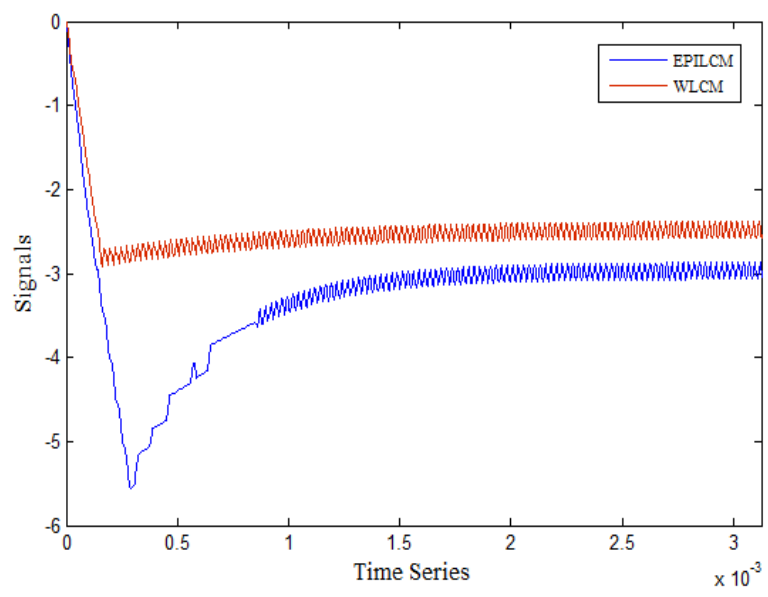

Fig 4. Comparison of propagation delay rate

The insecurity of message time delay is the main issue of synchronization error. The synchronization error is estimated by the total time delay of messages in the sensor network. The synchronization error calculation is based on variation of the clock parameter values. The input values are randomly taken from the time intervals. The synchronization error calculation in EPILCM protocol is based on the packet transmission from cluster member to base station. Fig 5 shows the synchronization error rate comparisons of EPILCM and 
WLCM protocols. The clock synchronization error rate obtain based on clock invariance using Eq. 21. The EPILCM synchronization error signal value ranges from 0 to $-1.1602 \mathrm{e}^{-}$ ${ }^{04}$ and WLCM synchronization error signal value ranges from 0 to $1.1602 \mathrm{e}^{-04}$.

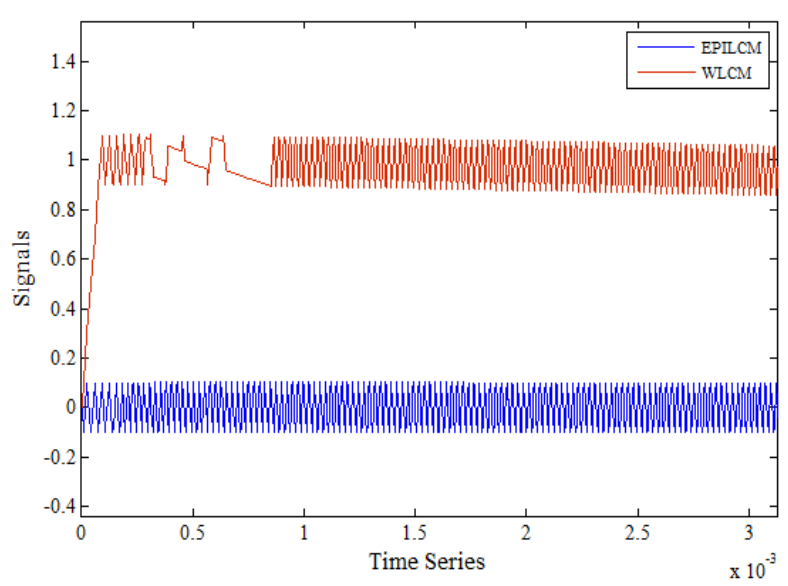

Fig 5. Comparison of clock synchronization error rate

Clock offset parameter value is calculated based on the clock inaccuracy. Fig 6 shows the clock offset rate comparisons of EPILCM and WLCM protocols. The EPILCM clock offset signal value ranges from 0 to $-28630 \mathrm{e}^{-}$ ${ }^{04}$ and WLCM clock offset signal value ranges from 0 to $7.1574 \mathrm{e}^{-04}$. It is observed that EPILCM protocol dynamically reduced the clock offset due to the usage of proportional techniques in EPI protocol.

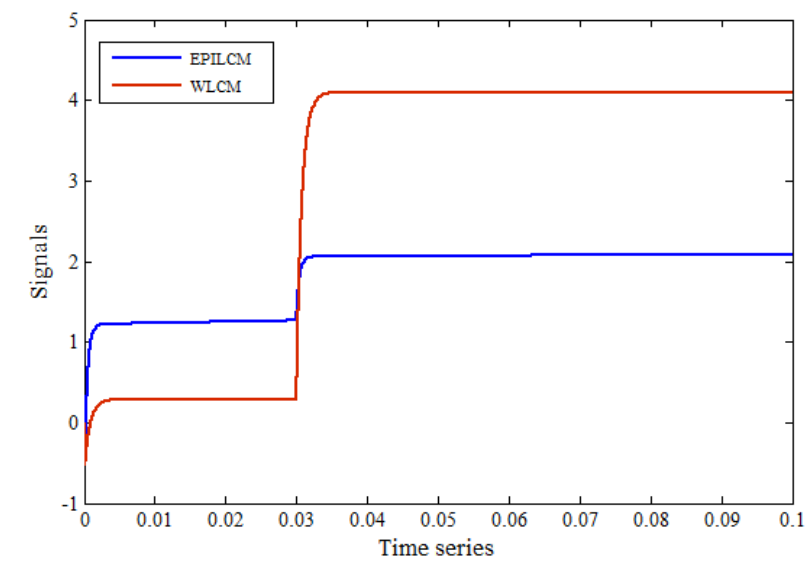

Fig 6. Comparison of clock offset rate

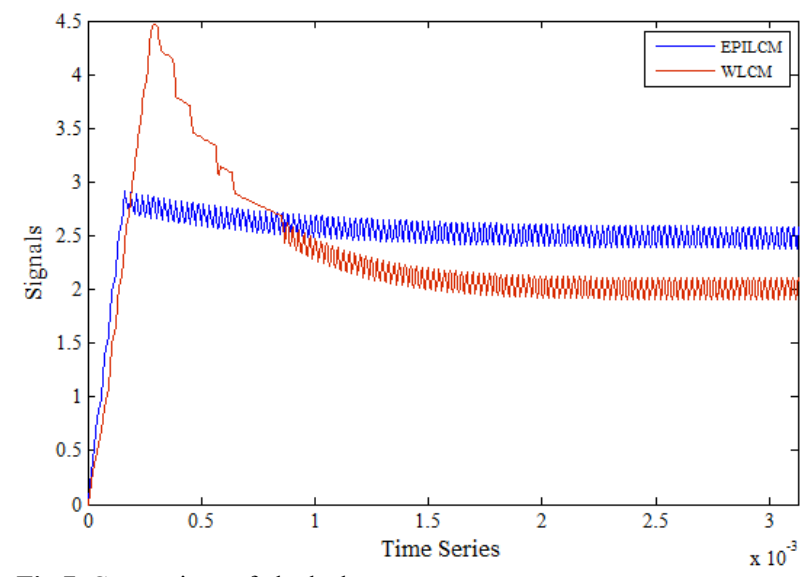

Fig 7. Comparison of clock skew rate
The clock frequency difference between the synchronizing nodes is called clock skew. Clock skew rate is calculated based on the clock frequency invariance. Fig 7 shows the clock skew rate comparisons of EPILCM and WLCM protocols. In proposed technique EPI method is compensating the clock skew value. The EPILCM clock skew signal value ranges from 0 to $1.1602 \mathrm{e}^{-04}$ and WLCM signal value clock skew ranges from 0 to $2.3204 \mathrm{e}^{-04}$. It is observed that EPILCM protocol reasonably reduced the clock skew due to the usage of integral techniques in EPI protocol. The clock parameter inaccuracy is calculated based on varying the clock skews invariance rate.

EPILCM protocol achieves clock synchronization time by transmitting $\mathrm{N}$ number of packets. Fig 8 shows the communication overhead comparisons of EPILCM and WLCM protocols. The EPILCM communication overhead signal value ranges from 0 to $1.8990 \mathrm{e}^{-04}$ and WLCM communication overhead signal value ranges from 0 to $2.1100 \mathrm{e}^{-04}$. The ranges shows EPILCM communication overhead is less compared to WLCM. The EPILCM protocol reasonably reduced the communication overhead due to the usage of proportional and integral techniques in EPILCM protocol.

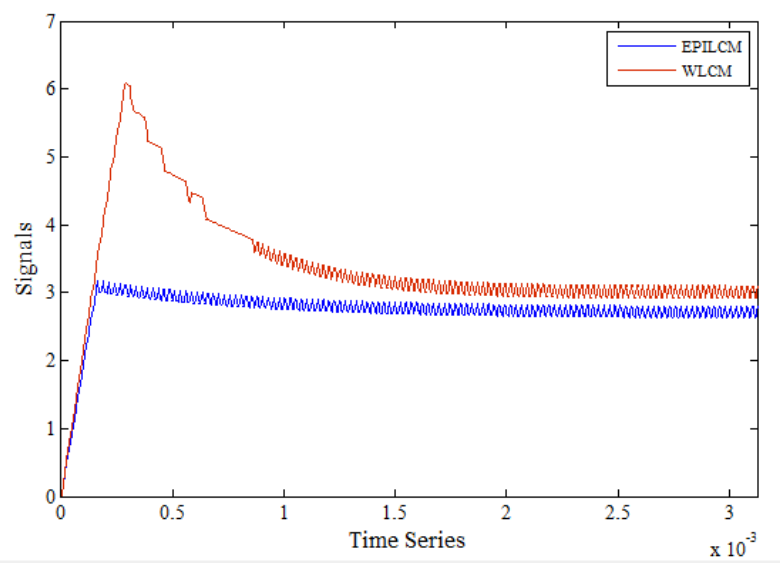

Fig 8. Comparison of communication overheads

The simulation results describe the performances of EPILCM and WLCM protocols. The compared results are obtained for the time interval. The EPILCM protocol reduces the synchronization error, propagation delay, communication overheads, and clock parameter inaccuracy than the WLCM protocol. The proposed EPILCM protocol provides the efficient synchronization over the networks.

\section{Conclusion}

Routing and synchronization scheme is the energy consumption critical issues in WSNs. The proposed EPILCM protocol for clock synchronization is a consensus energy based method, where the sensor nodes are synchronized based on the EPI and ELCM method. Based on the routing technology, synchronization was carried at two levels. At the first level, the clock parameter time will be compensating for synchronization. At second level consensus based synchronization method was used for efficient synchronization. EPILCM protocol is used to reduce the synchronization error, propagation delay, and communication overhead among the sensor nodes. The WLCM protocol for clock synchronization is developed based on weight. EPILCM and WLCM are compared and result shows EPILCM outperforms WLCM. Further research would be focused on calculating energy consumption rate and clock 
inaccuracy rate for both intra cluster and inter cluster routing for efficient synchronization and for achieving better accuracy in clock parameters.
This is an Open Access article distributed under the terms of the Creative Commons Attribution License

\section{References}

1. Ankit Aggarwal, and Mahesh Kumar, A Survey of Routing Protocols in Wireless Sensor Network, International Journal of Advanced Research in Computer Science and Software Engineering, Volume 5, Issue 6, pp. 269 -272, June 2015.

2. Archana Chauhan and Praveen Sharma, A Review on Routing Protocols For Wireless Sensor Network, International Journal of Science, Engineering and Technology Research (IJSETR), Volume 4, Issue 6, pp. 2004 - 2008, June 2015.

3. Pratishtha Parganiha and Kakelli Anil Kumar, An Energy-Efficient Clustering with Hybrid Coverage Mechanism (EEC-HC) in Wireless Sensor Network for Precision Agriculture, JOURNAL OF Engineering Science and Technology Review, Volume 11, Issue 3 pp. 97 - 103, July 2018.

4. Awadia Ahmed Ali, Guo Yanling and Chang Zifan, Study of Hall Effect Sensor and Variety of Temperature Related Sensitivity, J. Eng. Technol. Sci., Vol. 49, No. 3, pp. 308-321, July 2017.

5. Shashank Bholane, Devendrasingh Thakore, Time Synchronization in Wireless Sensor Net-works, International Journal of Scientific \& Engineering Research Volume 3, Issue 7, pp. 01 -06, July 2012.

6. Nikolaos M. Freris, Scott R. Graham, and P. R. Kumar, Fundamental Limits on Synchronizing Clocks Over Networks, IEEE Transactions On Automatic Control, Vol. 56, No. 6, pp. 1352 - 1364 , June 2011.

7. BrunoMarques and ManuelRicardo, Synchronization of applicationdriven WSN, EURASIP Journal on Wireless Communications and Networking, pp. 1- 22, 2017.

8. Lin Lin, Shiwei Ma and Maode Ma, A Group Neighborhood Average Clock Synchronization Protocol for Wireless Sensor Networks, Sensors, pp. 14744-14764, August 2014.

9. Habib Aissaoua, Makhlouf Aliouat, Ahcène Bounceur, and Reinhardt Euler, A Distributed Consensus based Clock Synchronization Protocol for Wireless Sensor Networks, Wireless Personal Communications, Springer Verlag, pp. 1 - 25, March 2017,

10. Enrique Mallada, Xiaoqiao Meng, Michel Hack, Li Zhang, and Ao Tang, Skewless Network Clock Synchronization Without Discontinuity: Convergence and Performance, IEEE/ACM Transactions on Networking,Vol.23, No.5, pp. 1619-1633, October 2015.

11. Kasım Sinan Yıldırım, Ruggero Carli, and Luca Schenato, Adaptive Proportional-Integral Clock Synchronization in Wireless Sensor Networks, IEEE Transactions On Control Systems Technology, pp. 1- 14, 2017.

12. XuWang, DanielJeske and ErchinSerpedin, An Overview of a Class of Clock Synchronization Algorithms for Wireless Sensor Networks:
A Statistical Signal Processing Perspective, Algorithms 2015, pp. 590-620 August 2015.

13. Leandro Tavares Bruscato, Tales Heimfarth 2 and Edison Pignaton de Freitas, Enhancing Time Synchronization Support in Wireless Sensor Networks, Sensor, pp. 1 -18, December 2017.

14. Enrique Mallada, Member,IEEE, Xiaoqiao Meng, Michel Hack, Li Zhang, and Ao Tang, Skewless Network Clock Synchronization Without Discontinuity: Convergence and Performance, IEEE/ ACM Transactions On Networking, Vol.23, No.5, pp. 1619 - 1633, October 2015.

15. Bharath Sundararaman, Ugo Buy, and Ajay D. Kshemkalyani, Clock synchronization for wireless sensor networks: a survey, Elsevier, Ad Hoc Networks, pp. 281-323, January 2005.

16. Zhaobin Liu, Qiang Ma, and Wenzhi Liu, Victor S. Sheng, and Liang Zhang and Gang Liu, Access Control Model Based on Time Synchronization Trust in Wireless Sensor Networks, Sensors, pp. 0115, June 2018.

17. Kan Xiea, Qianqian Cai, and Minyue $\mathrm{Fu}, \mathrm{A}$ fast clock synchronization algorithm for wireless sensor networks, Elsevier, Automatica, pp. 133 - 142, September 2018.

18. Zhaowei Wang, Peng Zeng, Linghe Kong, Dong Li and Xi Jin, Node-Identification-Based Secure Time Synchronization in Industrial Wireless Sensor Networks, Sensors, pp.1- 18 August 2018.

19. Kyoung - Lae Noh, Yik- Chung Wu, Khalid Qaraqe, and Bruce W.Suter, Extension of Pairwise Broadcast Clock Synchronization for Multi cluster Sensor Networks, EURASIP Journal on Advances in Signal Processing Volume 2008, Article ID 286168, pp. 1 -10, November 2007.

20. WenlunYang and MinyueFu, A proportional integral estimatorbased clock synchronization protocol for wireless sensor networks, ISA Transactions, pp. 1-13, March 2017.

21. Jianshe $\mathrm{Wu}$, Licheng Jiao, and Ranran Ding, Average time synchronization in wireless sensor networks by pairwise messages, Computer Communications, pp. 221-233, Septemper 2011.

22. Nikhath Tabassum, Geetha D. Devanagavi, and Rajashekhar C. Biradar, Clock synchronization in wireless sensor network using least common multiple, Int. J. Electron. Commun. (AEU), pp. $446-$ 457, October 2017.

23. M.Muthumalathi, Dr. P.B.Pankajavalli, Mobility-Zonal based Stable Election Routing Protocol for Wireless Sensor Networks, IPASJ International Journal of Computer Science (IIJCS), Volume 6, Issue 10, pp. 1- 9, October 2018. 